\title{
Conexões
}

\section{Protocolos de avaliação da coordenação motora para pessoas com deficiência intelectual}

Aline Miranda Strapasson ${ }^{1}$ Gabriela Simone Harnisch ${ }^{1}$ Simone Thiemi Kishimoto ${ }^{1}$

\section{RESUMO}

A avaliação da coordenação motora de pessoas com deficiência intelectual (DI) é foco de diversas pesquisas na área da Educação Física. Neste sentido, o objetivo do presente estudo é constatar protocolos de avaliação da coordenação motora para pessoas com DI, além de apresentar os protocolos de avaliação da coordenação motora encontrados, com maior enfoque no Teste do Movimento ABC (M-ABC Test), pois o mesmo foi desenvolvido especificamente para avaliar pessoas com a referida deficiência. Foram utilizadas as bases de dados Scirus, Sciverse, Scielo, Embase, Pubmed, Lilacs e Google Scholar. Constatou-se que a literatura traz vários testes que elucidam a avaliação da coordenação motora em pessoas com DI, porém, ainda há a necessidade de maior aplicação. Concluiu-se que a avaliação motora é uma forma de fornecer subsídios para que os professores de Educação Física possam orientar suas práticas conforme as necessidades envolvidas na população em foco, além de fornecer dados consistentes para estudos científicos.

Palavras-Chave: Avaliação. Educação física. Protocolos. Coordenação motora. Deficiência intelectual.

\footnotetext{
${ }^{1}$ Universidade Estadual de Campinas

Submetido em: 02 jun.2016

Aceito em: 31 maio 2017

Contato: aline-strapasson@ @otmail.com

Conexões: Educ. Fís., Esporte e Saúde, Campinas: SP, v. 15, n. 3, p. 272-292, jul./set. 2017. ISSN: 1980-9030
} 


\section{The assessment of motor coordination for people with intellectual disability}

\section{A BStraCT}

The assessment of motor coordination for people with intellectual disability (ID) is the focus of numerous research in the Physical Education area. In this sense, the objective of this study was to verify the assessments protocols for motor coordination for people with ID, as well as present all protocols for assessing motor coordination that we have found, focusing specially on the Movement ABC Test (M-ABC Test), because it was developed specifically to evaluate people with ID. In order to achieve these goals, we selected scientific publications at the following databases: Scirus, SciVerse, Scielo, Embase, PubMed, Lilacs and Google Scholar. It was found that literature provides several tests that elucidate the assessment of motor coordination in people with ID, however, there is still a need for wider application. We concluded that the motor evaluation is a way of providing support for Physical Education teachers so they can guide their practices according to the needs of the population in question, and that it also provides consistent data for scientific studies.

Keywords: Evaluation, Physical education. Protocols. Motor coordination. Intellectual disability.

\section{Protocolos de evaluación de la coordinación motora para personas con discapacidad intelectual}

\section{RESUMEN}

La evaluación de las habilidades motoras de las personas con discapacidad intelectual (DI) es el enfoque de varios estudios en el área de Educación Física. En este sentido, el objetivo de este estudio fue examinar los protocolos de evaluación de la coordinación de las personas con DI, y presentar los protocolos de evaluación de la coordinación que han sido encontrados, con mayor énfasis en la batería de evaluación de Movimiento $\mathrm{ABC}$ (Test $\mathrm{M}-\mathrm{ABC}$ ), porque fue desarrollado específicamente para evaluar las personas con este tipo de discapacidad. Las bases de datos que se utilizaron: Scirus, SciVerse, Scielo, Embase, Pubmed, Lilacs y Google Scholar. Se encontró que la literatura aporta varios tests que aclaran la evaluación de la coordinación motora en los individuos con DI, todavía hay aun necesidad de más aplicación. Se concluyó que la evaluación motora es una forma de proporcionar apoyo a los profesores de Educación Física para que conduzcan sus prácticas de acuerdo con las necesidades de la población en cuestión, y que puede proporcionar datos consistentes para estudios científicos. 
Palabras Clave: Evaluación. Educación física. Protocolos. Coordinación motora. La discapacidad intelectual. 


\section{INTRODUÇÃO}

A criação de diversas leis que visam à participação ativa de pessoas com deficiência (PD) na sociedade, tem incitado profissionais de todas as áreas a buscar conhecimento, a respeito de atuação para o desenvolvimento de um trabalho adequado e de qualidade para esta população (FERNANDES; SCHLESE; MOSQUERA, 2011).

Sabe-se que existe uma mobilização para que as PD, de diferentes tipos, possam ter uma vida mais ativa e participativa na sociedade, por meio de procedimentos de inclusão que estimulam a autonomia e independência (FERNANDES; SCHLESE; MOSQUERA, 2011; RIBEIRO et al., 2014).

$\mathrm{Na}$ área da saúde, e de forma mais específica na Educação Física (EF), encontrase uma gama de informações a respeito de metodologias do processo de ensino e aprendizagem envolvendo PD, ou seja, formas adequadas de ministrar aulas, maneiras de agir, materiais para utilizar, bem como as adaptações à serem realizadas. Informações necessárias e relevantes para a atuação na EF junto a diferentes tipos de deficiências são discutidas por diversos autores da área (ADAMS et al., 1985; FREITAS; CIDADE, 1997; SOUZA, 1994; WINNICK, 2004; GORGATTI; COSTA, 2005; MAUERBERG DE CASTRO, 2005; RODRIGUES, 2006; TEIXEIRA, 2008; DIEHL, 2008).

Entre os diferentes tipos encontra-se a Deficiência Intelectual (DI), um dos focos deste estudo, que é designada, dentre outros aspectos, com funcionamento intelectual abaixo da média (SAVELSBERGH et al., 2000). Geralmente, na DI ocorrem atrasos do desempenho e da percepção motora, além de limitações significativas no comportamento (ELLIOTT; BUNN, 2004). As desordens motoras em pessoas com DI incluem dificuldades na introdução e execução dos movimentos, com significativa lentidão e problemas no controle de membros (SAVELSBERGH et al., 2000; ELLIOTT; BUNN, 2004).

Essas características motoras devem ser avaliadas pelos professores de EF para que possa ser gerado um programa de intervenção adequado. Nesse sentido, ressalta-se a importância de estimular e promover o desenvolvimento motor para que o aluno chegue o mais próximo possível do padrão normal de desenvolvimento (BIANCONI, 2012).

Gorla, Rodrigues e Araújo (2009), na área da avaliação motora para PD enfatizam a relevância de avaliar os alunos para verificar se as aulas ministradas estão proporcionando benefícios ou não. 
A avaliação serve a um objetivo muito importante na área do desenvolvimento motor (GORLA et al., 2009). Quando realizada em vários aspectos do comportamento motor de um indivíduo, torna possível ao especialista em EF Adaptada (EFA) monitorar alterações desenvolvimentistas, identificar atrasos e obter esclarecimentos sobre estratégias instrutivas (GORLA et al., 2009).

Diante do exposto, o objetivo principal do presente estudo é averiguar protocolos de avaliação da coordenação motora para pessoas com DI. Como objetivos específicos, apresentam-se os protocolos de avaliação da coordenação motora encontrados na busca, bem como, apresentar e explicar o Teste $M-A B C$, a fim de fornecer subsídios para professores de EF em suas atuações profissionais.

\section{A Coordenação Motora}

A coordenação motora tem sido alvo de várias investigações e pesquisas, diante das preocupações e necessidades crescentes de um entendimento mais claro e profundo sobre sua relação direta com a formação corporal da criança, bem como sobre sua associação com a qualidade do movimento e com a melhoria do desenvolvimento motor (BALLESTERO, 2008).

A coordenação de movimentos, de acordo com a idade, é entendida como uma interação harmoniosa e econômica de músculos, nervos e sentidos, com a finalidade de produzir ações cinéticas, precisas e equilibradas (motricidade voluntária), e reações rápidas e adaptadas às situações (motricidade reflexa) (KIPHARD, 1976).

Também pode ser entendida como a base do movimento homogêneo e eficiente, que exige uma extensa organização do sistema nervoso, com utilização dos músculos certos, no tempo certo e intensidade correta, sem gastos energéticos (RAUCHBACH, 1990; VIDAL et al., 2009), ou como a capacidade para realizar grandes movimentos, utilizando o corpo inteiro, envolvendo os grandes músculos do corpo (PEREIRA, 2001).

Isso implica na harmonia dos movimentos voluntários dos grandes segmentos corporais ou na capacidade de controle dos atos motores, que colocam em ação todo o corpo (LE BOULCH, 1987). São os movimentos naturais como rolar, engatinhar, andar, correr, pular, saltar, saltitar, dentre outros (LE BOULCH, 1987).

Em termos biomecânicos, a coordenação motora se refere à ordenação dos impulsos de força em uma ação motora, ordenação de acontecimentos em relação a dois ou mais eixos perpendiculares (MEINEL, 1987). Quanto aos fatores fisiológicos, 
relaciona-se com as leis que regulam os processos de contração muscular entre agonistas e antagonistas, bem como os processos nervosos que lhe são subjacentes (MEINEL, 1987). Já o aspecto pedagógico, tem ligação ordenada com as fases de um movimento ou com ações parciais (MEINEL, 1987).

Uma interação perfeita (coordenação) tem que satisfazer as seguintes condições:

1) a adequada medida de força, que determina a amplitude e velocidade do movimento;

2) a adequada utilização dos músculos, que influi na condução e orientação do movimento;

3) a capacidade em alternar rapidamente entre tensão e relaxamento musculares, premissa de toda forma de adaptação motora (MEINEL, 1987).

A coordenação motora é necessária em todos os movimentos que as pessoas realizam , variando apenas o grau de solicitação (SILVA, 1998). Quanto melhor for a qualidade da coordenação, mais fácil e precisamente será realizado o movimento (CATENASSI et al., 2007). A realização do movimento se torna mais flexível e econômico, de modo que decresce o consumo energético e, consequentemente, a capacidade máxima de oxigênio cresce em relação a uma determinada solicitação muscular, baixando simultaneamente o nível de fadiga (SILVA, 1998; CATENASSI et al., 2007).

Desordens de coordenação motora de uma PD, como por exemplo, uma criança com DI, são apresentadas de modos bem distintos ao serem comparadas com uma desordem de coordenação de uma criança sem deficiência (CATENASSI et al., 2007). Na DI há um déficit muito grande, relacionado ao esquema e imagem corporal, dificultando a realização dos movimentos (FRUG, 2001). Além disso, dependendo do tipo de DI, observamos um grande comprometimento do equilíbrio, lateralidade, motricidade global, organização temporal e espacial (FRUG, 2001).

Existem diferentes métodos para avaliar o nível de competência motora e, eventualmente, de estabelecer um diagnóstico (GORLA; RODRIGUES; ARAÚJO). Um teste que avalia as competências motoras tem que satisfazer às condições de fidelidade e validade, assim como apresentar tabelas relativas às diferentes idades (GORLA; RODRIGUES; ARAÚJO).

É preciso conhecer muito bem os diversos instrumentos de aferição e seus propósitos educacionais, além de aplicá-los com as técnicas adequadas, interpretar os resultados corretamente e propor uma intervenção (GORLA; RODRIGUES; ARAÚJO; JOHNSON, 1972; FARIA JUNIOR, 1986). 
O estudo da coordenação motora se reveste, assim, de grande importância em diversas disciplinas científicas, como a aprendizagem motora, o controle motor e o desenvolvimento motor (LOPES et al., 2003). Essas disciplinas focam os seus esforços no sentido de entender como as ações motoras se processam em diferentes níveis, desde a forma como são reguladas até o seu resultado (LOPES et al., 2003).

\section{Avaliação Motora}

No processo de elaboração de programas de EF, muitos são os fatores que influenciam para que seu sucesso se efetive, dentre eles a avaliação (MACHADO, 2012).

A avaliação é um recurso que se aplica ao avaliado e ao processo, pode ser um indicador quantitativo ou qualitativo e utilizar elementos objetivos ou subjetivos empregados para comparação de resultados (MACHADO, 2012). Não deve ser encarada como produto de um momento, mas sim de um período, o que possibilita reajustar o programa a fim de atingir o objetivo almejado (MACHADO, 2012).

Para a avaliação, há uma gama de itens importantes que precisam ser observados, como: testes orientados para o produto (examinam o resultado de determinada habilidade); testes orientados para o processo (examinam o padrão da performance); testes que usam normas como referência (comparam as habilidades da criança às de outros sujeitos de idade e gênero similares); testes que usam critérios como referência (examinam a qualidade do movimento); fidedignidade (testes que fornecem valores consistentes de um período de teste a outro); e validade (testes que medem o que realmente afirmam medir) (GALLAHUE; OZMUN; GODWAY, 2013).

A objetividade, a simplicidade e a "fidedignidade" são muito importantes no processo de avaliação (GORLA et al., 2009). Cabe frisar que, para efeito de comparações futuras, toda avaliação e reavaliação deve seguir o mesmo processo metodológico e, se possível, reproduzir igualmente as condições ambientais, de instrumento, de avaliadores e demais parâmetros importantes (GALLAHUE; OZMUN; GODWAY, 2013). Além disso, deve-se ter atenção em relação às condições (ambiente) em que os indivíduos serão testados, o treinamento do avaliador e a relação entre número de avaliados e tempo para a avaliação (GORLA et al., 2009; GALLAHUE; OZMUN; GODWAY, 2013; MELLO, PASSETO, 2008).

No processo de avaliação, o teste visa identificar o desempenho do avaliado em uma variável qualquer, a medida quantifica o desempenho observado por meio do teste e a avaliação julga a medida referenciada a uma norma ou critério pré-estabelecido (COSTA E SILVA; GORLA; COSTA, 2012). 
Assim, são muitos os testes existentes e cada um tem um objetivo específico. Dentre eles, os testes motores são classificados como uma prova específica, que permite medir determinada característica de uma pessoa, que pode ser comparada com a de outros indivíduos (ROSA NETO, 2002). Prova motora, é um conjunto de atividades determinadas para uma idade a fim de indicar o avanço ou atraso motor de uma criança (ROSA NETO, 2002). O conjunto de provas e de testes para avaliar várias características motoras de um indivíduo é denominado de Bateria Motora (ROSA NETO, 2002).

Entende-se, assim, que avaliações se tornam relevantes para que sejam evidenciadas as necessidades da população e podem ter diferentes características e peculiaridades (ROSA NETO, 2002). Dentre as diversas possibilidades, destacam-se as avaliações aplicadas às PD (ELLIOTT; BUNN, 2004).

A avaliação motora para PD pode ser utilizada para colocar os alunos em programas que irão otimizar o seu aprendizado, para determinar se os indivíduos precisam ser avaliados com mais critérios, identificar os pontos fortes e fracos dos avaliados, além de permitir um direcionamento para o desenvolvimento das metas e objetivos dos programas de EF (ELLIOTT; BUNN, 2004).

\section{A Deficiência Intelectual}

O Censo Demográfico de 2010 apontou que existem 2.611.536 pessoas com Deficiência Intelectual (DI) no Brasil (INSTITUTO, 2010). A Associação Americana de Deficiência Intelectual e Desenvolvimento (AAIDD) (AMERICAN, 2011) caracteriza a DI como limitações significativas no funcionamento intelectual, acompanhado de comprometimentos no comportamento adaptativo, expresso nas habilidades sociais, práticas e conceituais.

E, ainda, a American Associationon Mental Retardation (AAMR) considera a DI como uma condição deficitária, que envolve: habilidades intelectuais; comportamento adaptativo; participação comunitária; interações e papéis sociais; condições etiológicas e de saúde; aspectos contextuais, ambientais, culturais; e as oportunidades de vida do sujeito (CARVALHO; MACIEL, 2003).

As limitações no funcionamento cognitivo e no desempenho de tarefas, como as de comunicação, cuidado pessoal e de relacionamento social provocam maior lentidão na aprendizagem e no desenvolvimento (CARVALHO; MACIEL, 2003). A forma como pessoas com DI percebem o mundo e os objetos; reflete na comunicação, na socialização, na autonomia, na consciência de si própria, na compreensão e na resolução de problemas (ALVES et al., 2008). Apresentam dificuldades em perceber as 
informações relevantes do meio, em memorizar, em exprimir-se ou imaginar novos conceitos. Geralmente necessitam de mais tempo para aprender a falar, a andar e a assimilar competências básicas para cuidar de si, tal como vestir-se ou comer com autonomia (ALVES et al., 2008).

A DI caracteriza-se por um desenvolvimento cognitivo mais lento e por uma diferença na forma como as estruturas cognitivas são aplicadas (ALBUQUERQUE, 1996).

As principais manifestações da DI situam-se na menor eficiência em situações de aprendizagem, de aquisição de aptidões e de resolução de problemas. Sua definição implica três conceitos chave (ALBUQUERQUE, 1996). O primeiro, deficiência/dificuldade, refere-se ás limitações que colocam o indivíduo em desvantagem quando funciona em sociedade (BELO et al., 2008). O segundo conceito, a inteligência, envolve a capacidade para pensar, planejar, resolver problemas, compreender e aprender. Por fim, o comportamento adaptativo, representa as competências conceituais, práticas e sociais que as pessoas aprendem para serem capazes de funcionar no cotidiano (BELO et al., 2008).

Quanto mais elevado for o grau da deficiência intelectual, maior será o atraso no desenvolvimento como um todo (LOPES; SANTOS, 2002). Porém, para que um indivíduo seja diagnosticado com DI, as limitações mencionadas acima devem ocorrer durante o período de desenvolvimento até os 18 anos de idade (AMERICAN, 2011).

Tratando-se das causas da DI, a lista dos fatores de risco segue dois direcionamentos: tipos de fatores de risco e momento da ocorrência (AMERICAN, 2006). De acordo com a AAIDD, dentre os tipos de fatores de risco, existem quatro grupamentos:

1) Biomédicos: relacionados aos processos biológicos;

2) Sociais: fatores que estão relacionados com a interação social e familiar;

3) Comportamentais: fatores relacionados a comportamentos prejudiciais por parte materna e,

4) Educacionais: relacionados à disponibilidade de apoio educacional (AMERICAN, 2006).

Reportando-se a prevenção, existem três níveis:

1) Pré-natal: que envolve a prevenção da condição que, do contrário, poderia resultar em DI;

Peri-natal: que envolve ações para prevenir que uma condição existente possa causar DI; 
3) Pós-natal: que envolve ações para minimizar a gravidade dos impedimentos funcionais associados à etiologia (AMERICAN, 2006).

Esses novos conceitos preocupam-se com as peculiaridades e habilidades individuais, baseando-se nas áreas do comportamento adaptativo (FERREIRA, 1997). Desta forma, torna-se necessário avaliar os vários aspectos do comportamento adaptativo, descrito como a eficiência ou o grau em que um indivíduo atende aos padrões de independência pessoal e responsabilidade social esperados de sua idade e grupo social (AMERICAN, 2006; FERREIRA, 1997).

Os autores supracitados complementam que o comportamento adaptativo possui várias áreas específicas, perfazendo um total de dez áreas. São elas: comunicação, cuidado pessoal, vida doméstica, aptidões sociais, participação comunitária, saúde e segurança, funções acadêmicas, auto direção, lazer e trabalho (AMERICAN, 2006; FERREIRA, 1997). Além disso, a avaliação deve ser contínua e dinâmica, inserindo observações sistemáticas dos profissionais que atuam com o indivíduo avaliado (AMERICAN, 2006; FERREIRA, 1997).

As pessoas com DI podem apresentar defasagens nos aspectos psicomotor, cognitivo e afetivo-social fundamentais para o desenvolvimento das características comportamentais, educacionais e a independência nas atividades diárias (HAMILTON, 2002). Por conta dessas defasagens, elas podem ser rejeitadas por seus colegas em brincadeiras e jogos por não apresentarem competência suficiente nos movimentos (HAMILTON, 2002).

É importante frisar que as pessoas com DI, de modo algum, constituem um grupo homogêneo quanto ao comportamento, função intelectual, habilidades físicas, níveis de desenvolvimento e outras características pertinentes (GORLA; ARAÚJO, 2007).

Portanto, cabe ao professor de EF fornecer subsídios e suportes para a participação efetiva de pessoas com DI nas aulas, a partir dos conhecimentos obtidos por meio de avaliações prévias e objetivas.

\section{Estudos encontrados}

A avaliação motora em crianças e adolescentes com DI é necessária para a intervenção de qualidade, porém, se faz necessário identificar claramente os critérios que o instrumento oferece (GORLA et al., 2009). O Quadro 1 apresenta os testes encontrados através das bases: Scirus, Sciverse, Scielo, Embase, Pubmed, Lilacs e Google Scholar. 
Quadro 1 - Protocolos de avaliação da coordenação motora

\begin{tabular}{|c|c|c|c|}
\hline Protocolos & Autores & Variável & População \\
\hline $\begin{array}{l}\text { Teste de habilidades de } \\
\text { crianças e jovens }\end{array}$ & Griffiths & $\begin{array}{l}\text { Habilidades motoras, } \\
\text { locomotoras, coordenação } \\
\text { motora fina e a performance }\end{array}$ & $\begin{array}{l}\text { Crianças e jovens do } \\
\text { nascimento aos } 8 \text { anos de } \\
\text { idade }\end{array}$ \\
\hline $\begin{array}{l}\text { Teste de desenvolvimento } \\
\text { motor grosso }\end{array}$ & Ulrich & $\begin{array}{l}\text { Habilidade motora geral e o } \\
\text { desenvolvimento motor total }\end{array}$ & $\begin{array}{l}\text { Crianças de } 3 \text { a } 10 \text { anos de } \\
\text { idade }\end{array}$ \\
\hline $\begin{array}{l}\text { Escala de desenvolvimento do } \\
\text { comportamento da criança: o } \\
\text { primeiro ano de vida }\end{array}$ & $\begin{array}{l}\text { Pinto; } \\
\text { Vilanova; } \\
\text { Vieira }\end{array}$ & $\begin{array}{l}\text { Desenvolvimento do } \\
\text { comportamento (motor e de } \\
\text { atividade) }\end{array}$ & $\begin{array}{l}\text { Crianças com diferentes } \\
\text { patologias com o } \\
\text { referencial de normalidade } \\
\text { (Idade: de } 1 \text { a } 12 \text { meses) }\end{array}$ \\
\hline $\begin{array}{l}\text { Teste motor Milestone } \\
\text { (TDMMT) }\end{array}$ & $\begin{array}{l}\text { Burton; } \\
\text { Miller }\end{array}$ & $\begin{array}{l}\text { Movimento por meio de } \\
\text { intervenções orientadas }\end{array}$ & $\begin{array}{l}\text { Crianças com profunda } \\
\text { deficiência múltipla }\end{array}$ \\
\hline $\begin{array}{l}\text { Escala de desenvolvimento } \\
\text { motor Peabody (PDMS) }\end{array}$ & $\begin{array}{l}\text { Folio; } \\
\text { Fewell }\end{array}$ & $\begin{array}{l}\text { Habilidades motoras de } \\
\text { locomoção, reflexo, } \\
\text { manipulação de objetos e } \\
\text { movimento estático }\end{array}$ & $\begin{array}{l}\text { Crianças com deficiência } \\
\text { intelectual } \\
\text { (do nascimento aos } 6,9 \\
\text { anos de idade) }\end{array}$ \\
\hline $\begin{array}{l}\text { Escala de desenvolvimento } \\
\text { motor (EDM) }\end{array}$ & Rosa Neto & $\begin{array}{l}\text { Coordenação motora global e } \\
\text { fina; equilíbrio; esquema } \\
\text { corporal; lateralidade; } \\
\text { organização espacial e } \\
\text { temporal }\end{array}$ & $\begin{array}{l}\text { É indicado para avaliar } \\
\text { atrasos no } \\
\text { desenvolvimento de } \\
\text { crianças }\end{array}$ \\
\hline $\begin{array}{l}\text { Teste de Bruininks-Oseretsky } \\
\text { de proficiência motora } \\
\text { (BOTMP) }\end{array}$ & $\begin{array}{l}\text { Bruininks; } \\
\text { Bruininks }\end{array}$ & $\begin{array}{l}\text { Coordenação motora fina; } \\
\text { coordenação dos membros } \\
\text { superiores; equilíbrio e } \\
\text { coordenação bilateral; força e } \\
\text { agilidade }\end{array}$ & $\begin{array}{l}\text { Crianças com deficiência } \\
\text { intelectual entre } 4,5 \text { a } 14,5 \\
\text { anos }\end{array}$ \\
\hline $\begin{array}{l}\text { Bateria de avaliação de } \\
\text { movimento para crianças - } \\
\text { Teste do movimento ABC } \\
\text { (M-ABC Test) }\end{array}$ & $\begin{array}{l}\text { Henderson; } \\
\text { Sugden; } \\
\text { Barnett }\end{array}$ & $\begin{array}{l}\text { Habilidades motoras fina e } \\
\text { grossa que englobam } \\
\text { habilidades manuais de } \\
\text { destreza, mira,captura e } \\
\text { equilíbrio }\end{array}$ & $\begin{array}{l}\text { Crianças e adolescentes } \\
\text { entre } 3 \text { a } 16 \text { anos }\end{array}$ \\
\hline $\begin{array}{l}\text { Avaliação da coordenação } \\
\text { motora grossa (BGMA) }\end{array}$ & $\begin{array}{l}\text { Hughes; } \\
\text { Riley }\end{array}$ & $\begin{array}{l}\text { Habilidades motoras de } \\
\text { movimento fundamental e de } \\
\text { movimentos especializados }\end{array}$ & $\begin{array}{l}\text { Crianças e adolescentes } \\
\text { entre } 5,5 \text { a } 12,5 \text { anos }\end{array}$ \\
\hline $\begin{array}{l}\text { Teste de coordenação } \\
\text { corporal para crianças (KTK) }\end{array}$ & $\begin{array}{l}\text { Gorla; } \\
\text { Rodrigues; } \\
\text { Araújo }\end{array}$ & $\begin{array}{l}\text { Coordenação motora total e o } \\
\text { domínio corporal }\end{array}$ & $\begin{array}{l}\text { Crianças e adolescentes } \\
\text { com deficiência intelectual } \\
\text { entre } 4,5 \text { a } 14,5 \text { anos }\end{array}$ \\
\hline
\end{tabular}

A partir dos dados apresentados do Quadro 1, optou-se por expor maiores detalhes acerca da Bateria de Avaliação de Movimento para crianças - Teste do Movimento ABC (Movement ABC Test ou M-ABC Test), por ser um teste desenvolvido especificamente para avaliar pessoas com DI, validado e com pouca utilização no Brasil.

\section{Teste M-ABC}

Para Henderson e Sugden (1992); Sugden e Wright (1998), crianças com dificuldades de movimento têm sido foco de atenção de pesquisadores há várias décadas, nas mais diversas áreas do conhecimento e intervenção. No entanto, pode-se destacar que mais recentemente tem havido uma preocupação especial de vários pesquisadores em relação ao diagnóstico de crianças com dificuldades de movimento. 
Mais especificamente, o Teste M-ABC, que tem sido objeto de muitas pesquisas (HENDERSON; SUGDEN, BARNETT, 2007; HENDERSON; SUGDEN, 1992).

O Teste M-ABC foi elaborado por Henderson e Sugden (1992). Trata-se de um teste composto por uma bateria de testes motores envolvendo habilidades manuais, de equilíbrio e com bola e, também, uma lista de checagem que possibilita a identificação de crianças de 3 a 16 anos de idade com dificuldades de movimento. Esse teste foi validado com uma população de 1.234 crianças americanas (HENDERSON; SUGDEN, BARNETT, 2007; HENDERSON; SUGDEN, 1992).

É composto por oito itens em cada uma das quatro faixas etárias. As provas estão organizadas em habilidades com bola, destreza manual, equilíbrio estático e dinâmico. Conforme os grupos as provas são diferentes, porém avaliam as mesmas habilidades motoras (HENDERSON; SUGDEN, 1992). Uma criança com boa coordenação e inteligência média pode completar o teste em 20-40 minutos (SMITS-ENGELSMAN; NIEMEIJER; WAELVELDE, 2011).

O Quadro 2 sugerido por Henderson e Sudgen (1992), apresenta as provas do Teste $\mathrm{M}-\mathrm{ABC}$.

Quadro 2 - Provas do Teste M-ABC

\begin{tabular}{|c|c|c|}
\hline Habilidade Motora & Tarefa & Critérios de Exclusão \\
\hline Destreza Manual & $\begin{array}{l}\text { Colocar } 12 \text { pinos em uma prancha de } \\
\text { madeira }\end{array}$ & $\begin{array}{l}\text { Pegar mais de um pino de uma só vez ou utilizar as } \\
\text { duas mãos }\end{array}$ \\
\hline Destreza Manual & $\begin{array}{l}\text { Enfiar um cordão em vários furos } \\
\text { localizados em uma prancha de } \\
\text { costura }\end{array}$ & $\begin{array}{l}\text { Errar a sequência lógica de passar o cordão nos } \\
\text { furos, esquecer de passar o cordão em algum dos } \\
\text { furos ou trocar de mão }\end{array}$ \\
\hline Destreza Manual & $\begin{array}{l}\text { Contornar pontos tracejados em } \\
\text { formato de uma flor }\end{array}$ & $\begin{array}{l}\text { Inverter a direção no qual traceja, retirar o lápis e } \\
\text { colocar fora do ponto onde parou. Deve ser } \\
\text { considerado o número de vezes que a criança saiu do } \\
\text { limite das duas linhas e permaneceu por uma } \\
\text { distância de no mínimo } 1 / 2 \text { polegada }\end{array}$ \\
\hline Habilidades com a Bola & $\begin{array}{l}\text { Quicar e pegar a bola de tênis com } \\
\text { uma mão } \\
\text { *cada mão } 10 \text { tentativas }\end{array}$ & $\begin{array}{l}\text { Quicar a bola com uma mão e pegar com as duas } \\
\text { Quicar a bola com uma mão e pegar com a outra } \\
\text { Quicar a bola com uma mão e pegar com o auxílio } \\
\text { do corpo }\end{array}$ \\
\hline Habilidades com a Bola & $\begin{array}{l}\text { Arremessar o saquinho de feijão } \\
\text { dentro da caixa (distância de } 2 \\
\text { metros) }\end{array}$ & $\begin{array}{l}\text { Pisar ou ultrapassar a linha limite para o arremesso. } \\
\text { Arremessar o saquinho } \\
\text { de feijão com as duas mãos }\end{array}$ \\
\hline Equilíbrio estático & $\begin{array}{l}\text { Equilíbrio em uma perna (equilíbrio } \\
\text { da cegonha) } \\
* 20 \text { segundos }\end{array}$ & $\begin{array}{l}\text { Mover o pé de equilíbrio do local original } \\
\text { Afastar o pé que estava no joelho contrário } \\
\text { Tirar as mãos da cintura }\end{array}$ \\
\hline Equilíbrio Dinâmico & $\begin{array}{l}\text { Saltar com os pés juntos por dentro } \\
\text { dos quadrados }(45 \mathrm{~cm}) \text { consecutivos }\end{array}$ & $\begin{array}{l}\text { Aterrissar fora do quadrado ou em cima das linhas } \\
\text { Aterrissar com os pés muito distantes um do outro }\end{array}$ \\
\hline Equilíbrio Dinâmico & $\begin{array}{l}\text { Caminhar sobre a linha }(4,5 \text { metros } \\
\text { de comprimento), um pé em frente ao } \\
\text { outro }\end{array}$ & $\begin{array}{l}\text { Deixar espaço entre os dedos e o calcanhar } \\
\text { Pisar fora da linha }\end{array}$ \\
\hline
\end{tabular}

Fonte: HENDERSON; SUDGEN, 1992.

Pellegrini, Souza Neto e Higara (2006) também citam as provas do Teste M-ABC:

1. Destreza manual:

1.1 Deslocando fileiras de pinos; 
1.2 Rosqueando porcas no parafuso;

1.3 Traçado da flor.

2. Habilidades com bola:

2.1 Recepção da bola com as duas mãos;

2.2 Lançando o saco de feijão na caixa.

3. Equilíbrio estático e dinâmico:

3.1 Equilíbrio em uma perna sobre uma placa;

3.2 Saltando pelos quadrados;

3.3 Equilíbrio da bola.

A utilização do Teste M-ABC deve seguir quatro etapas distintas: identificação; exploração clínica e intervenção de planejamento; avaliação do programa; e como ferramenta de pesquisa (HENDERSON; SUGDEN, 1992).

A identificação é utilizada para classificar as dificuldades de movimento de leve a moderado, sendo possível identificar uma criança com dificuldade motora, comparando a pontuação da mesma com os dados normativos (HENDERSON; SUGDEN, 1992). A exploração clínica e intervenção para o planejamento ocorrem para definir como ajudar a criança que tem dificuldade motora (HENDERSON; SUGDEN, 1992). Essa tarefa requer uma estratégia diferente do que simplesmente estabelecer a dificuldade que existe. $\mathrm{O}$ examinador deve pensar além do perfil de competências motoras da criança, para incluir todos os outros aspectos relevantes do seu desenvolvimento (por exemplo, se a criança está ou não com algum problema de atenção ou problema de desenvolvimento motor) (HENDERSON; SUGDEN, 1992).

$\mathrm{O}$ Teste $\mathrm{M}-\mathrm{ABC}$ fornece ao examinador a oportunidade de gravar muitas características diferentes do comportamento de uma criança de uma forma sistemática. Com as informações fornecidas pelo teste um programa de intervenção individualizado pode ser desenvolvido, especificando os objetivos a curto e longo prazo (HENDERSON; SUGDEN, 1992).

Waelvelde et al. (2004) indicam que o manual original do Teste M-ABC permite a diferenciação de grupos de controle, crianças com transtorno de aprendizagem e crianças prematuras. Entretanto, alguns estudos relatados apresentam baixa correlação com o Bruininks-Oseretsky Test of Motor Proficiency (BRUININKS, 2005), e com o KTK (RAUCHBACH, 1990). Esse dado, no entanto, pode ser entendido hipoteticamente pela data de padronização dos testes, já o Teste M-ABC foi desenvolvido aproximadamente 20 anos após os outros com que foi comparado, e todos esses estão sob uma ótica ecológica, ou seja, avaliam habilidades que podem ser treinadas ou não em determinadas condições, não se atendo a perfis neuromaturacionais.

O Teste M-ABC já está estabelecido como uma ferramenta de pesquisa. Ele é utilizado para: selecionar os temas de estudos experimentais; como instrumento de 
avaliação nos estudos longitudinais de crianças com desordens de coordenação motora; como meio de estudar os fatores que contribuem para a baixa competência motora; e como uma medida de mudança em estudos de intervenção (HENDERSON; SUGDEN, 1992).

Quanto à validade do teste, foi realizado um estudo no qual participaram 133 crianças de 7-9 anos e foram aplicados dois testes de habilidades com bola e dois de equilíbrio estático e dinâmico. Para a realização dos testes as crianças foram divididas em dois grupos, sendo 107 crianças que realizaram a bateria para 7-8 anos e 26 que realizaram a bateria para grupos de 9-10 anos.

Nos testes de habilidades com bola, 90 crianças foram identificadas com algum grau de dificuldade de movimento, enquanto que 43 crianças apresentaram desenvolvimento "normal". Os resultados apresentados mostraram que a análise entre os testes com bola e os de equilíbrio estático e dinâmico variaram de não significante para uma correlação altamente significativa, com coeficiente de $-0,74$. A partir deste estudo observou-se validade concorrente para o Teste M-ABC (WAELVELDE et al., 2004).

Em outra pesquisa (WAELVELDE et al., 2007), realizada três anos depois, foram fornecidas evidências da confiabilidade do Teste M-ABC. Nesse estudo, 33 crianças (24 meninos e 9 meninas), com idades entre 4-5 anos, já diagnosticadas anteriormente com mau desempenho motor, foram testadas. Os resultados encontrados indicaram que o Teste M-ABC apresentou uma medida confiável de identificação das dificuldades de movimento em crianças (WAELVELDE et al., 2007).

A segunda versão do Teste denominado M-ABC-2 foi desenvolvida anos mais tarde (HENDERSON; SUGDEN, BARNETT, 2007). Nessa, houve inclusão de uma extensão da faixa etária e a redução do número de faixas etárias, denominadas bandas, bem como uma revisão das tarefas. As bandas são três: AB1, para crianças de 3-6 anos, AB2, de 7-10 anos e AB3, de 11-16 anos (HENDERSON; SUGDEN, 2007).

Schulz et al. (2011) se propuseram a realizar um estudo sobre a validade das provas do M-ABC-2 separadas por categorias, isto é, especificamente a de destreza manual, habilidades de captura com bola e equilíbrio. Foram avaliadas 1.172 crianças (566 meninos e 606 meninas) com idade entre 3-16 anos. Destas, 431 crianças encontravam-se na faixa etária de 3-6 anos (AB1), 333 crianças na faixa etária de 7-10 anos (AB2) e 408 crianças na faixa etária de 11-16 anos (AB3). O estudo demonstrou que as modificações validadas no $\mathrm{M}-\mathrm{ABC}-2$ são recorrentes, e que futuramente os trabalhos deverão concentrar-se na validade estrutural do teste em crianças com dificuldade de movimento, com o qual o M-ABC é geralmente utilizado (SCHULZ et al., 2011). 
Na DI, o teste M-ABC foi utilizado por Rockenbach (2014), que realizou um estudo de caso com duas crianças, sendo com Síndrome de Aspenger e outra com Mielomeningocele, ambas com 12 anos de idade. Foi identificado neste estudo que os testes apresentaram resultado satisfatório, porém, há uma necessidade de adaptação de materiais e da faixa etária na realização do teste para esta população (ROCKENBACH, 2014).

Um estudo realizado nos EUA (BORREMANS; RINTALA; MCCUBBIN, 2010), avaliou 30 adolescente com Síndrome de Aspenger (SA) e comparou com o grupo controle, foram avaliadas a aptidão física, atividade física, força muscular, velocidade de corrida, resistência cardiorrespiratória e coordenação motora através do teste $\mathrm{M}-\mathrm{ABC}$, foi identificado que o grupo com SA, são menos ativos e apresentaram escores menores em todos os testes em comparação ao grupo controle (BORREMANS; RINTALA; MCCUBBIN, 2010).

Duronjic e Valkova (2010) avaliaram 5 crianças (5 e 6 anos de idade) com a Síndrome do Espectro Autista, foi utilizado o teste M-ABC para a avaliação da coordenação motora, pré e pós programa de intervenção, realizado 2 vezes por semana, com duração de 2 meses. Neste estudo, foi identificado que todas as crianças obtiveram melhora no teste (DURONJIC; VALKOVA, 2010).

Percebe-se que testes como o M-ABC são essenciais se quisermos chegar a uma melhor compreensão de como ajudar as crianças com dificuldades motoras. Uma das exigências de tais estudos é uma ferramenta de avaliação válida e confiável para documentar as mudanças no desempenho motor de crianças e adolescentes participantes com e sem deficiência intelectual.

Observamos que apesar do Teste M-ABC ser bastante utilizado em estudos no Brasil, sua padronização para esta população ainda não foi realizada, apesar de ser sugerido em diversos estudos (SOARES; DE MARCO, 2012; MAGALHÃES et al. (2009).

\section{CONSIDERAÇÕES Finais}

Ressalta-se que pesquisas relacionadas à avaliação motora de pessoas com DI vêm sendo foco para diversos estudos na área da EF, porém, ainda existe a necessidade de novos estudos, principalmente no que tange a validação dos mesmos para populações brasileiras.

Ainda, enfatiza-se que a realização da avaliação motora para com alunos com DI serve também para que professores de EF possam direcionar suas intervenções conforme a necessidade que cada aluno possui, e por meio destas, possam ser 
evidenciadas melhoras no âmbito da coordenação motora. Portanto, é relevante a avaliação motora para que haja uma melhor compreensão de como ajudar crianças com desordens de coordenação motora.

Para tanto, recomenda-se a utilização dos testes citados neste artigo, pois os mesmos fornecem parâmetros válidos e fidedignos para a realização de avaliações e pesquisas científicas.

Nesse sentido, sugere-se a realização de avaliações da coordenação motora para pessoas com DI, bem como, que as mesmas sejam publicadas para que sirvam como subsídios para os demais profissionais que enfocam a DI em seus trabalhos e estudos.

\section{REFERÊNCIAS}

ADAMS, Ronald C. et al. Jogos, esportes e exercícios para o deficiente físico. 3. ed. São Paulo: Manole, 1985.

ALBUQUERQUE Maria Cristina Petrucci de. A criança com deficiência mental ligeira. 1996. Tese (Doutorado em Psicologia e Ciências da Educação) - Universidade de Coimbra, Coimbra, 1996.

ALVES, Fabiola et al. As TIC nas dificuldades intelectuais e desenvolvimentais. Diversidades, Madeira, v. 6, n. 22, p. 25-27, out./dez. 2008.

AMERICAN ASSOCIATION ON INTELLECTUAL AND DEVELOPMENTAL DISABILITIES (AAIDD). Definition of intellectual disability. Washington, 2011.

Disponível em: <http://aaidd.org/intellectual-disability/definition/faqs-on-intellectualdisability\#.UzF_fldVLc>. Acesso em: 05 jun. 2015.

AMERICAN ASSOCIATIONON MENTAL RETARDATION (AAMR). Retardo mental: definição, classificação e sistemas de apoio. 10. ed. Porto Alegre: Artes Médicas, 2006.

BALLESTERO, Carmem Lucia Guimarães. Avaliação da coordenação motora, ideias fundamentais e investigação empírica a partir da bateria de testes KTK. 2008. Dissertação (Mestrado) - Universidade do Porto, Porto, 2008.

BELO, Chantal et al. Deficiência intelectual: terminologia e conceptualização. Diversidades, Madeira, v. 6, n. 22, p. 4-9, out./dez. 2008. 
BIANCONI, Elizabeth de Cássia. Influência de um programa de educação física no desenvolvimento psicomotor de jovens e adultos com deficiência intelectual. 2012. Dissertação (Mestrado em Ciências Humanas) - Universidade Estadual de São Carlos, São Carlos, 2012.

BRUININKS, Robert H.; BRUININKS, Brett. Bruininks-oseretskytest of motor proficiency. $2^{\text {nd }}$ ed. Minneapolis: Pearson Assessment, 2005.

BORREMANS, Erwin; RINTALA, Pauli; MCCUBBIN, Jeffrey A. Physical fitness and physical activity in adolescents with Asperger Syndrome: a comparative study. Human Kinetics Journals, Champaign, v. 27, n. 4, p. 308-320, oct. 2010.

BURTON, Allen William; MILLER, Daryl E. Top-down motor milestone test. In: BURTON, Allen William (Ed.). Movement skill assessment. Champaign: Human Kinetics, 1998.

CARVALHO, Erenice Natália Soares; MACIEL, Diva Maria Moraes de Albuquerque. Nova concepção de deficiência mental segundo a American Association on Mental Retardation - AAMR: sistema 2002. Temas em Psicologia, Ribeirão Preto, v. 11, n. 2, p. 147-156, 2003.

CATENASSI, Fabrizio Zandonadi et al. Relação entre índice de massa corporal e habilidade motora grossa em crianças de quatro a seis anos. Revista Brasileira de Medicina do Esporte, São Paulo, v. 13, n. 4, p. 227-230, jul./ago. 2007.

COSTA e SILVA, Anselmo Atahyde; GORLA, José Irineu; COSTA, Leonardo Trevisan. Avaliação motora em esporte adaptado. In: MACHADO, Alexandre Fernandes; ABAD, César Cavinato Cal (Ed.). Manual de avaliação física. São Paulo: Ícone, 2012.

DIEHL, Rosilene Moraes. Jogando com as diferenças. 2. ed. São Paulo: Phorte, 2008.

DURONJIC, Milica; VÁLKOVÁ, Hana. The influence of early intervention movement programs on motor skills development in preschoolers with autism spectrum disorder (case studies). Acta Universitatis Palackianae Olomиcencis, Gymnica, Olomouc, v. 40, n. 2, p. 37-45, 2010.

ELLIOTT, Digby; BUNN, Lindsay. Motor disorders in children with intellectual disabilities. In: DEWEY, Deborah; TUPPER, David E. (Ed.). Developmental motor disorders: a neuropsychological perspective. New York: Guilford, 2004.

FARIA JUNIOR, Alfredo Gomes de. Fundamentos pedagógicos I: avaliação em educação física. Rio de Janeiro: Ao Livro Técnico, 1986. 
FERNANDES, Lorena Barolo; SCHLESENER, Anita; MOSQUERA, Carlos. Breve histórico da deficiência e seus paradigmas. Revista do Núcleo de Estudos e Pesquisas Interdisciplinares em Musicoterapia, Curitiba, v. 2, p. 132-144, 2011.

FERREIRA, Ana Isabel de Figueiredo. Proposta de avaliação motora para a pessoa deficiente mental, após estudo de caso realizado nas APAEs da região de Campinas. 1997. Tese (Doutorado em Educação Física) - Universidade Estadual de Campinas, Campinas, 1997.

FOLIO, M. Rhonda; FEWELL, Rebecca R. Peabody developmental motor scales. $2^{\text {nd }}$ ed. Austin: PRO-ED, 2000.

FREITAS, Patricia Silvestre; CIDADE, Ruth Eugênia Amarante. Noções sobre educação física e esporte para pessoas portadoras de deficiências: uma abordagem para professores de $1^{\circ}$ e $2^{\circ}$ graus. Uberlândia: Gráfica Breda, 1997.

FRUG, Chystianne Simões. Educação motora em portadores de deficiência. São Paulo: Plexus, 2001.

GALLAHUE, David L.; OZMUN, John C.; GOODWAY, Jacqueline D. Compreendendo o desenvolvimento motor: bebês, crianças, adolescentes e adultos. 7 . ed. Porto Alegre: Artmed, 2013.

GORGATTI, Marcia Greguol; COSTA, Roberto Fernandes da. Atividade física adaptada. São Paulo: Manole, 2005.

GORLA, José Irineu; ARAÚJO, Paulo Ferreira de. Avaliação motora em educação física adaptada: teste KTK para deficientes mentais. São Paulo: Phorte, 2007.

GORLA, José Irineu; RODRIGUES, José Luiz; ARAÚJO, Paulo Ferreira de. Avaliação motora em educação física adaptada: teste KTK. 2. ed. São Paulo: Phorte, 2009.

GORLA, José Irineu et al. Fundamentos da avaliação motora em educação física adaptada. Revista Digital EFdeportes, Buenos Aires, v. 13, n. 128, 2009. Disponível em: <http://www.efdeportes.com/efd128/fundamentos-da-avaliacao-motora-emeducacao-fisica-adaptada.htm>.

GRIFFITHS, Ruth. The abilities of young children: a comprehensive system of mental measurement for the first eigth years of life. London: Child Development Research Centre, 1970.

HAMILTON, Sylvester Sutton. Evaluation of clumsiness in children. American Family Physician, Kansas City, v. 66, n. 8, p. 1435-1440, oct. 2002. 
HENDERSON, Sheila E.; SUGDEN, David A. Movement assessment battery for children. London: Psychological Corporation, 1992.

HENDERSON, Sheila E.; SUGDEN, David A.; BARNETT, Anna L. Movement assessment battery for children. $2^{\text {nd }}$ ed. London: Pearson, 2007.

HUGHES, Jeanne E.; RILEY, Ann. Basic gross motor assessment: tool for use with children having minor motor dysfunction. Physical Therapy, Alexandria, v. 61, n. 4, p. 503-511, 1981.

INSTITUTO BRASILEIRO DE GEOGRAFIA E ESTATÍSTICA (IBGE). População e tipo de deficiência. Brasília, 2010. Disponível em:

<http://biblioteca.ibge.gov.br/visualizacao/periodicos/93/cd_2010_caracteristicas_popul acao_domicilios.pdf>. Acesso em: 05 jun. 2015.

JOHNSON, Prescott K. Evaluatión del rendimento físico em los programas de educación física. Buenos Aires: Paidós, 1972.

KIPHARD, Ernest Jonny. Insuficiências del movimiento y de coordenacíon em edad de $1^{a}$ escuela primaria. Buenos Aires: Kapelusz, 1976.

LE BOULCH, Jean. Educação psicomotora: psicocinética na idade escolar. Porto Alegre: Artmed, 1987.

LOPES, Vitor Pires et al. Estudo do nível de desenvolvimento da coordenação motora da população escolar (6 a 10 anos) da região autônoma dos Açores. Revista Portuguesa de Ciência do Desporto, Porto, v. 3, n. 1, p. 47-60, 2003.

LOPES, Vitor Pires; SANTOS, Manuela Zita Bentes dos. Desenvolvimento das habilidades motoras básicas em crianças portadoras de deficiência intelectual. Revista Digital EFdeportes, Buenos Aires, v. 8, n. 50, jul. 2002.

MACHADO, Alexandre Fernandes. Critérios científicos aplicados na educação física. In: MACHADO, Alexandre Fernandes; ABAD, César Cavinato Cal (Ed.). Manual de avaliação física. São Paulo: Ícone, 2012.

MAGALHÃES, Lívia C. et al. Análise comparativa da coordenação motora de crianças nascidas a termo e pré-termo, aos 7 anos de idade. Revista Brasileira de Saúde Materno Infantil, Recife, v. 9, n. 3, p. 293-300, set. 2009.

MAUERBERG de CASTRO, Eliane. Atividade física adaptada. São Paulo: Tecmed, 2005. 
MEINEL, Kurt. Motricidade I: teoria da motricidade esportiva sob o aspecto pedagógico. Rio de Janeiro: Livros Técnicos e Científicos, 1987.

MELLO, Marco Túlio de; PASETTO, Carolina Ventura Fernandes. Protocolos para avaliação física e fisiológica em pessoas com deficiência. In: GORGATTI, Marcia Greguol; COSTA, Roberto Fernandes da (Org.). Atividade física adaptada: qualidade de vida para pessoas com necessidades especiais. 2. ed. Barueri: Manole, 2008.

PELLEGRINI, Ana Maria; SOUZA NETO, Samuel; HIGARA Chyntia et al. Dificuldades motoras em crianças de 9-10 anos de idade: seriam os meninos mais descoordenados? Rio Claro, 2006. Disponível em:

<http://www.unesp.br/prograd/PDFNE2006/artigos/capitulo1/dificuldades.pdf>

PEREIRA, Carlise Olschowsky. Estudos dos parâmetros motores em crianças de 02 a 06 anos de idade no município de Cruz Alta - RS. 2001. Dissertação (Mestrado em Ciências do Movimento Humano) - Universidade Estadual de Santa Catarina, Florianópolis, 2001.

PINTO, Elizabeth Batista; VILANOVA, Luiz Celso Pereira; VIEIRA, Raymundo Manno. O desenvolvimento do comportamento da criança: o primeiro ano de vida: padronização de uma escala para a avaliação e o acompanhamento. São Paulo: Casa do Psicólogo: FAPESP, 1997.

RAUCHBACH, Rosemary. Atividade física para terceira idade. Curitiba: Lovise, 1990.

RIBEIRO, Aline Pereira et al. Cenário da inserção de pessoas com deficiência no mercado de trabalho: revisão sistemática. Revista da Universidade Vale do Rio Verde, Três Corações, v. 12, n. 2, p. 268-276, ago./dez. 2014.

ROCKENBACH, Joeli Andreia. Estratégias pedagógicas para uma avaliação motora adaptada: um relato de experiência. 2014. Trabalho de Conclusão de Curso (Graduação em Educação Física) - Universidade Federal do Rio Grande do Sul, Porto Alegre, 2014.

RODRIGUES, David. Atividade motora adaptada: a alegria do corpo. São Paulo: Artes Médicas, 2006.

ROSA NETO, Francisco. Manual de avaliação motora. Porto Alegre: Artmed, 2002.

SAVELSBERGH, Geert et al. Information-movement coupling in children with Down Syndrome. In: WEEKS, Daniel J.; CHUA, Romeo; ELLIOT, Digby (Ed.). Perceptualmotor behavior in Down Syndrome, Champaign: Human Kinetics, 2000. 
SCHULZ, Joerg et al. Structural validity of the movement ABC-2 test: factor structure comparisons across three age groups. Research in Developmental Disabilities, v. 32, n. 4, p. 1361-1369, jul./ago. 2011.

SILVA, Julio Cesar da. O treinamento e avaliação da coordenação em indivíduos da terceira idade. 1998. Trabalho de Conclusão de Curso (Graduação em Educação Física) - Universidade Estadual Paulista, Rio Claro, 1998.

SMITS-ENGELSMAN, Bouwien; NIEMEIJER, Anuschska S.; WAELVELDE, Hilde Van. Is the movement assessment battery for children. 2nd edition a reliable instrument to measure motor performance in 3 year old children? Research in Developmental Disabilities, v. 32, n. 4, p. 1370-1377, 2011.

SOARES, Daniela Bento; DE MARCO, Ademir. Protocolo de avaliação motora em crianças de até 7 anos de idade. 2012. Pesquisa de Iniciação Cientifica (Graduação em Educação Física) - Universidade Estadual de Campinas, Campinas, 2012.

SOUZA, Pedro Américo de. O esporte na paraplegia e tetraplegia. Rio de Janeiro: Guanabara Koogan, 1994.

TEIXEIRA, Luzimar. Atividade física adaptada e saúde: da teoria à prática. São Paulo: Phorte, 2008.

ULRICH, Dale. Test of gross motor development. Austin: PRO-ED, 1985.

VIDAL, Sonia Moreira et al. Construção de cartas científicas da coordenação motora de crianças dos 6 aos 11 anos da região autônoma dos Açores. Revista Portuguesa de Ciências do Desporto, Porto, v. 9, n. 1, p. 24-35, jan. 2009.

WAELVELDE, Van H. et al. Aspects of the validity of the movement assessment battery for children. Human Movement Science, Amsterdam, v. 23, n. 1, p. 49-60, jun. 2004.

WAELVELDE, Van H. et al. The reliability of the movement assessment battery for children for preschool children with mild to moderate motor impairment. Clinical Rehabilitation, London, v. 21, n. 1, p. 470-485, may 2007.

WINNICK, Joseph P. Educação física e esportes adaptados. São Paulo: Manole, 2004. 